\title{
Review of: "Using Integrated Multi-Omics Data Analysis to Identify 5-gene Signature for Predicting Survival of Patients with Hepatocellular Carcinoma"
}

\author{
Quqin $\mathrm{Lu}^{1}$ \\ 1 Nanchang University
}

Potential competing interests: The author(s) declared that no potential competing interests exist.

This study attempts to identify a reliable and effective gene signature related to the prognosis of HCC. The authors screened prognosis-related genes and those with SNP or CNV by bioinformatics methods, and verified the results. The topic of the study is very interesting. However, there are several points that should be considered to improve the manuscript.

1. If possible, you should present the characteristics or data types of the datasets that used in the study with Table.

2. What kind of treatment did HCC patients in the datasets receive? Hepatectomy, or RFA? The authors need to consider whether these patients can be evaluated together because the therapeutic curability of these two is different.

3. As for your results and conclusion, you describe the 5-gene signature model shows independent predictive performance. But according to the results of your multivariate analysis, both in the training and validation sets, your model works together with other clinical factors on the patients' OS. So, do you need to explain "independent predictive performance" and "independent of the clinical features" or change these statements?

4. Figure legends should be concise and representative. Please do not duplicate the manuscript content completely as a figure legend. Please modify all figure legends.

5. In the "Clinical independence of the 5-gene signature" section of the Results, line 6 and line 21, the tables you cited are Table 5 and Table 4, but they doesn't match your actual tables. Please check for similar errors in the full manuscript. 